\title{
Scorpion Sting Complicated by Atypical Posterior Reversible Encephalopathy A Case Report With Review of the Literature \\ F. Zahra Abakka ${ }^{1 *}$, A. El Masloumi ${ }^{1}$, I. Zouita $^{1}$, D. Basraoui ${ }^{1}$, H. Jalal ${ }^{1}$
}

${ }^{1}$ Radiology Department, Mother and Child Hospital, CHU Mohammed VI, Marrakech, Morocco

DOI: $10.36347 /$ sjmcr.2021.v09i04.030

| Received: 16.03.2021 | Accepted: 21.04.2021 | Published: 29.04.2021

*Corresponding author: F. Zahra Abakka

\section{Abstract}

Posterior reversible encephalopathy (PRES) is a clinico radiological syndrome, which manifests itself by headaches, confusion, consciousness disorders or during a sudden rise in blood pressure but can be seen in other circumstances, such as during an immune reaction or a systemic infection. Its mechanism is explained by a lesion of the blood-brain barrier leading to a reversible vasogenic edema. The scarcity of sympathetic innervation of the posterior cerebral territories explains its usual localization. We report the case of an 18 years old girl who presented with generalized tonic-clonic seizures following a scorpion bite, which yielded to Diazepam. An emergency brain MRI was performed, showing bilateral and symmetrical signal abnormalities of the posterior cortico-subcortical territories and basal ganglia, compatible with an atypical PRES syndrome. The diagnosis of PRES syndrome complicating a scorpion bite was retained.

Keywords: PRES syndrome, MRI, posterior localization.

Copyright $(\odot 2021$ The Author(s): This is an open-access article distributed under the terms of the Creative Commons Attribution 4.0 International License (CC BY-NC 4.0) which permits unrestricted use, distribution, and reproduction in any medium for non-commercial use provided the original author and source are credited.

\section{INTRODUCTION}

Posterior reversible encephalopathy syndrome, also known by the acronym PRES, is a radioclinical entity combining reversible central nervous system involvement with typical MRI or CT brain imaging. There is a great variability in the clinical presentation of this syndrome and imaging aspects are sometimes atypical. Management may therefore be delayed by diagnostic error or misdiagnosis. The diagnosis must be evoked on the basis of clinical circumstances and then confirmed by imaging [1].

\section{CASE RePORT}

This is an 18 years old girl who presented following a scorpion bite with an interval of 15 hours, persistent fever, diffuse abdominal pain and intense headaches; complicated by generalized tonicoclonic convulsive seizures yielding to Diazepam. The clinical examination showed a Glasgow score of 12 with no signs of focalization and a blood pressure of $180 / 110$ $\mathrm{mmHg}$.

An emergency MRI of the brain was performed (Fig-1), showing bilateral and symmetrical signal abnormalities in the posterior cortico-subcortical territories and basal ganglia, which is compatible with the atypical form of PRES syndrome.

The diagnosis of PRES syndrome complicating a scorpion bite was retained. The patient received symptomatic treatment associated with an antihypertensive infusion. The evolution is marked by the normalization of the blood pressure figures and the resumption of consciousness without sequels.
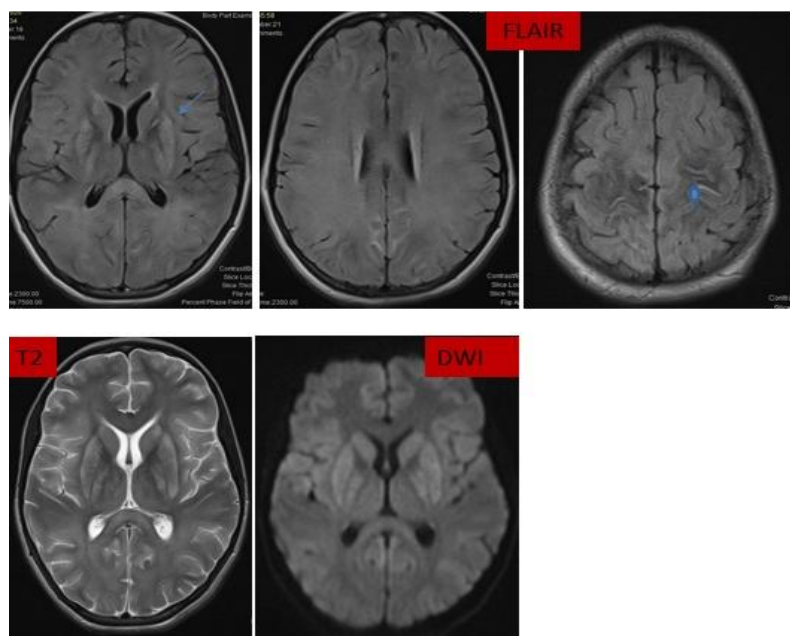

Fig-1: Brain MRI:T2, FLAIR AND DIFFUSION axial sequences: bilateral and symmetrical corticosteroid signal abnormalities under bilateral occipito parietal cortical, 
central gray nuclei and semi-oval centers in T2 hyper signal, Flair and diffusion (Blue arrow)

\section{DisCUSSION}

Posterior reversible encephalopathy syndromis a rare, poorly understood condition and probably remains underdiagnosed [2]. The worldwide incidence of RPE is unknown [3].

This condition is characterized by a prolonged but spontaneously reversible vasoconstriction of the cerebral arteries, occurring spontaneously or in particular circumstances [4]. Two theories remain the most accepted [5].

The first theory is based on the sequence of hypertension, cerebral autoregulation, hyper perfusion, and vasogenic cerebral edema due to extracapillary fluid leakage. The perivascular sympathetic innervation exerting a protective effect is richer in the anterior circulation and the carotid system, hence the posterior predominance of lesions.

The second theory is in favor of cerebral vasoconstriction secondary to arterial hypertension or to a systemic process. This autoregulatory phenomenon would lead to a decrease in cerebral perfusion and thus to cytotoxic edema. This theory is supported by the absence of (or minimal) blood pressure elevation in some cases. It argues more in favor of a systemic process (infection, preeclampsia, transplantation, anticancer chemotherapy) responsible for activation of the immune system and endothelial cells followed by endothelial damage with secondary hypoperfusion (systemic or regional).

Indeed, there are many circumstances that favor the occurrence of RPE. The most frequent are: arterial hypertension, eclampsia, pre-eclampsia; chemotherapy such as cyclophosphamide; chronic renal failure, dialysis and autoimmune diseases [1].

The clinical manifestations are varied [6]. They combine in a variable way four cardinal neurological symptoms: headache, seizures, consciousness disorders and visual disorders, these signs are most often accompanied by an acute and brutal increase in blood pressure.

Radiologically, reversible posterior encephalopathy is manifested by white matter and gray matter abnormalities preferentially affecting the posterior regions suggesting edema of the parietooccipital regions.

Imaging is essential and must be performed as soon as possible to allow early diagnosis in order to institute adequate treatment and limit the risk of irreversible lesions. On CT scan, the damage is marked by diffuse hypodensity. On MRI, the lesions appear in iso signal or hyposignal T1 and hypersignal T2 and
FLAIR. There is usually no enhancement after injection of contrast medium [1].

The typical appearance shows diffuse cortical, subcortical and deep lesions. The lesions are mainly located in the posterior regions: the parietal or occipital lobes are affected in $98 \%$ of cases. However, lesions also frequently affect the frontal lobes (68\%), the temporal lobes $(40 \%)$ and the cerebellar hemispheres (30\%) [7]. The bilateral and symmetrical nature of the lesions is very characteristic, although they are asymmetrical in $28 \%$ of cases.

Some radiological aspects are more atypical and should be known. More rarely, lesions may extend to the basal ganglia (14\%), brainstem (13\%) and deep white matter, including the splenium of the corpus callosum (10\%) [7]. Unilateral involvement and DCP should not lead to a rejection of the diagnosis

However, the clinical evolution is usually favorable with a more or less rapid improvement of the clinical signs and disappearance of the radiological images. Reversibility is usually complete, although there is a risk of neurological sequelae and even death [8].

\section{CONCLUSION}

Posterior reversible encephalopathy syndrom is a radio-clinical syndrome characterized by the association of variable neurological signs and white matter and gray matter abnormalities preferentially affecting the posterior regions. The evocative clinical context, as well as the rapidly reversible nature of the clinical and radiological abnormalities, suggest the existence of vasogenic cerebral edema related to a vasculopathy. Imaging, particularly MRI, plays an essential role in the diagnosis of this condition, which should be well known by radiologists and clinicians. Reversibility is conditioned by early diagnosis and correction of contributing factors. Moreover, knowledge of this syndrome should encourage the avoidance of unnecessary repeat imaging when the clinical course is favorable.

Conflicts of Interest: The authors declare no conflict of interest.

Authors' Contributions: All authors have read and approved the final version of the manuscript.

\section{REFERENCES}

1. Hugonnet E, Da Ines D, Boby H, Claise B, Petitcolin V, Lannareix V, Garcier JM. Posterior reversible encephalopathy syndrome (PRES): features on CT and MR imaging. Diagnostic and interventional imaging. 2013 Jan 1;94(1):45-52.

2. Wagih A, Mohsen L, Rayan MM, Hasan MM, AlSherif AH. Posterior reversible encephalopathy syndrome (PRES): restricted diffusion does not 
necessarily mean irreversibility. Polish journal of radiology. 2015;80:210-216.

3. Ducros A. Thunderclap headache. Rev Neurol (Paris). 2005; 161(6-7): 713-5.

4. Soo Y, Singhal AB, Leung T, Yu S, Mak H, Hao Q. Reversible cerebral vasoconstriction syndrome with posterior leuco encephalopathy after oral contraceptive pills. Cephalalgia. 2010; 30(1): 42-5.

5. Ural ÜM, Balik G, Şentürk Ş, Üstüner I, Çobanoğlu U, Şahin FK. Posterior reversible encephalopathy syndrome in a postpartum preeclamptic woman without seizure. Case reports in obstetrics and gynecology. 2014 Jan 30;2014:657903.
6. Postma IR. Long-term consequences of the posterior reversible encephalopathy syndrome in eclampsia and preeclampsia: a review of the obstetric and nonobstetric literature. Obstet Gynecol Surv. 2014; 69(5):287-300.

7. Bartynski WS, Boardman JF. Distinct imaging patterns and lesion distribution in posterior reversible encephalopathy syndrome. Am J Neuroradiol. 2007;28:1320-7.

8. Keyserling HF, Provenzale JM. Atypical imaging findings in a near-fatal case of posterior reversible encephalopathy syndrome in a child. AJR Am J Roentgenol. 2007;188:219-21. 